\title{
Síncope y paro cardiorrespiratorio, formas graves de presentación de la embolia pulmonar central. Reporte de tres casos clínicos
}

\author{
Dres. Gimena Loza, Victoria Briano, Agustina Perdomo, \\ Álvaro Niggemeyer, Juan Albistur
}

\begin{abstract}
Resumen
Palabras clave: TROMBOEMBOLIA PULMONAR SÍNCOPE

TRATAMIENTO TROMBOLÍTICO
\end{abstract}

El tromboembolismo pulmonar tiene una presentación clínica variada. Es fundamental tener un alto índice de sospecha para arribar al diagnóstico oportuno. El síncope se asocia a casos graves y tiene importancia pronóstica. El tratamiento trombolítico es la piedra angular en el subgrupo de pacientes de alto riesgo. Se presentan tres casos clínicos de tromboembolia pulmonar de alto riesgo con el fin de discutir el escenario clínico de presentación y el tratamiento instaurado.

\section{Syncope and cardiorespiratory arrest, severe forms of presentation of} central pulmonary embolism. Report of three clinical cases

\section{Summary}

Pulmonary thromboembolism has a varied clinical presentation. It is essential to have a high index of suspicion to arrive at a timely diagnosis. Syncope is associated with severe cases and is of prognostic significance. Thrombolytic treatment is the cornerstone in the subgroup of high-risk patients. Three clinical cases of high-risk pulmonary thromboembolism are presented in order to discuss the clinical presentation scenario and the established treatment.

Key words:

PULMONARY EMBOLISM

SYNCOPE

THROMBOLYSIS

Síncope e parada cardiorrespiratória, formas graves de apresentação de embolia pulmonar central. Relato de três casos clínicos

\section{Resumo}

O tromboembolismo pulmonar tem a presentação clínica variada. É essencial ter um alto índice de suspeita para chegar a um diagnóstico oportuno. A síncope está associada a casos graves e tem significado prognóstico. O tratamento trombolítico é a pedra angular no subgrupo de pacientes de alto risco. São apresentados três casos clínicos de tromboembolismo pulmonar de alto risco para discutir o quadro clínico e o tratamento instituído.

Palavras chave: TROMBOEMBOLISMO PULMONAR

SÍNCOPE

TRATAMENTO TROMBOLÍTICO

Centro Cardiovascular Universitario. Hospital de Clínicas, Universidad de la República. Montevideo, Uruguay.

Correspondencia: Dra. Gimena Loza Correo electrónico: gime.loza@gmail.com

Los autores declaran no tener conflictos de intereses .

Recibido Abr 9, 2021; aceptado Abr 27, 2021 


\section{Introducción}

La enfermedad tromboembólica venosa se puede presentar como tromboembolismo pulmonar (TEP) o trombosis venosa profunda (TVP). Es el tercer síndrome cardiovascular agudo en frecuencia luego del infarto de miocardio (IM) y del accidente cerebrovascular (ACV) y la tercera causa de muerte cardiovascular ${ }^{(1,2)}$.

Las múltiples formas de presentación y la inespecificidad de éstas han determinado que se lo denomine "el gran simulador". Se reporta que en menos del $10 \%$ se presenta como síncope o shock, pero se ha identificado TEP en hasta uno de cada seis pacientes hospitalizados por un primer episodio de síncope ${ }^{(3)}$. El diagnóstico y la intervención tempranos son primordiales, ya que la mayoría de las muertes por TEP agudo ocurren dentro de las primeras horas o días, y más del 70\% en la primera ho$\mathrm{ra}^{(4)}$. El tratamiento trombolítico tiene su lugar en los pacientes que se presentan con shock o hipotensión, y es una indicación de clase I, nivel de evidencia B, para estos pacientes ${ }^{(5)}$.

\section{Caso clínico 1}

Hombre de 77 años, dislipémico, trombosis venosa superficial en 2016, sin controles en salud. Consulta por episodio sincopal de breve duración, precedido de mareos. Días previos a la consulta, nota tumoración dolorosa en cara posterior de pierna izquierda, que desaparece el día de la consulta. Al ingreso, lúcido, presión arterial de 150/70 $\mathrm{mmHg}$, taquicardia de $120 \mathrm{cpm}$, taquipneico $26 \mathrm{rpm}$ saturando $97 \%$ al aire. En la figura $1 \mathrm{~A}$ se muestra el electrocardiograma (ECG) y en $1 B$ la radiografía de tórax (RxTx). De la paraclínica humoral se destaca: troponina ( $\mathrm{Tn}$ ) I: 0,85 ng/mL (percentil 99: 0,04 ng/mL), NT-proBNP: $1,266 \mathrm{pg} / \mathrm{mL}$. Gasometría arterial ventilando al aire: $\sin$ insuficiencia respiratoria, lactato de 2,1 $\mathrm{mmol} / \mathrm{L}$. Según el score de Ginebra original (9 puntos) ${ }^{(6)}$, y según el score de Wells simplificado (2 puntos) ${ }^{(7)}$, se trata de un TEP probable, por lo que se comienza anticoagulación precoz con heparina de bajo peso molecular (HBPM). Se realiza ecocardiograma transtorácico (ETT) que evidencia fracción de eyección del ventrículo izquierdo (FEVI): $60 \%$, hipertrofia concéntrica del VI, movimiento anormal del septum hacia el VI, sin otros trastornos sectoriales de la contractilidad, ventrículo derecho (VD) dilatado con función sistólica normal. Se realiza angiotomografía (angio TC) de arterias pulmonares (figura 1C), que confirma TEP central y dilatación del VD. Luego de la angio TC el paciente presenta deterioro hemodinámico con hipotensión arterial mantenida (a pesar de reposición con cristaloides), mala perfusión periférica y alteración del nivel de conciencia. Se administra alteplase (rTPA) a dosis de $10 \mathrm{mg}$ i/v bolo y luego $90 \mathrm{mg}$ i/v en BIC a pasar en dos horas. Se observa mejoría clínica y hemodinámica a la hora de infusión del fármaco, sin necesidad de vasopresores. Buena evolución posterior, otorgándose alta a domicilio a los diez días bajo tratamiento con rivaroxabán.

\section{Caso clínico 2}

Mujer, 65 años, obesa, portadora de hipertensión arterial (HTA). Ingresa por IM sin elevación del segmento ST, cinenangiocoronariografía (CACG) evidencia lesión severa de 3 vasos. Se realiza cirugía de revascularización miocárdica completa sin complicaciones. ETT previo al alta, FEVI de $45 \%$, cavidades derechas de dimensiones normales. Alta a domicilio a los siete días. Doce días luego del alta la paciente presenta episodio sincopal de reposo, precedido de palpitaciones rápidas y síndrome neurovegetativo (SNV) intenso que motiva consulta. $\mathrm{Al}$ ingreso, se encuentra polipneica (26 rpm), Sat $\mathrm{O}_{2} 92 \%$ al aire, normotensa, taquicardia de $120 \mathrm{cpm}$, ingurgitación yugular, sin edemas de miembros inferiores. En la figura $2 \mathrm{~A}$ se muestra el trazado ECG. De la paraclínica se destaca: TnI: $0,18 \mathrm{ng} / \mathrm{mL}$ (percentil 99: 0,04), RxTx con derrame pleural izquierdo leve. ETT: FEVI de 50\%, aurícula derecha (AD) severamente dilatada, VD severamente dilatado disfuncionante (TAPSE $12 \mathrm{~mm}$ ), gradiente VD/AD: 45 $\mathrm{mmHg}$, presión sistólica de la arteria pulmonar: 53 $\mathrm{mmHg}$, derrame pericárdico de $13 \mathrm{~mm}$ sobre la pared libre del VD (figura $2 \mathrm{~B}$ ). Se realiza angio TC que se muestra en la figura $2 \mathrm{C}$, y que confirma TEP central. Se inicia anticoagulación con HBPM. Se observa deterioro hemodinámico con elementos clínicos de bajo gasto, por lo que se decide a las 12 horas del ingreso realizar tratamiento fibrinolítico con alteplase ( $10 \mathrm{mg} \mathrm{i} / \mathrm{v}$ en bolo $+90 \mathrm{mg} \mathrm{i} / \mathrm{v}$ en dos horas), seguida de rápida mejoría desde el punto de vista hemodinámico y respiratorio. El seguimiento ecocardiográfico descarta complicación hemorrágica a nivel pericárdico y mejoría de los parámetros anatómicos y funcionales del VD (figura 2B), con disminución del gradiente VD-AD a $32 \mathrm{mmHg}$. Alta a domicilio al octavo día bajo tratamiento con apixabán (5 mg cada 12 horas).

\section{Caso clínico 3}

Hombre, 74 años, con antecedentes de insuficiencia venosa crónica de miembros inferiores, HTA, diabetes, obesidad, ex tabaquista y con enfermedad pulmonar obstructiva crónica (EPOC). Confina- 


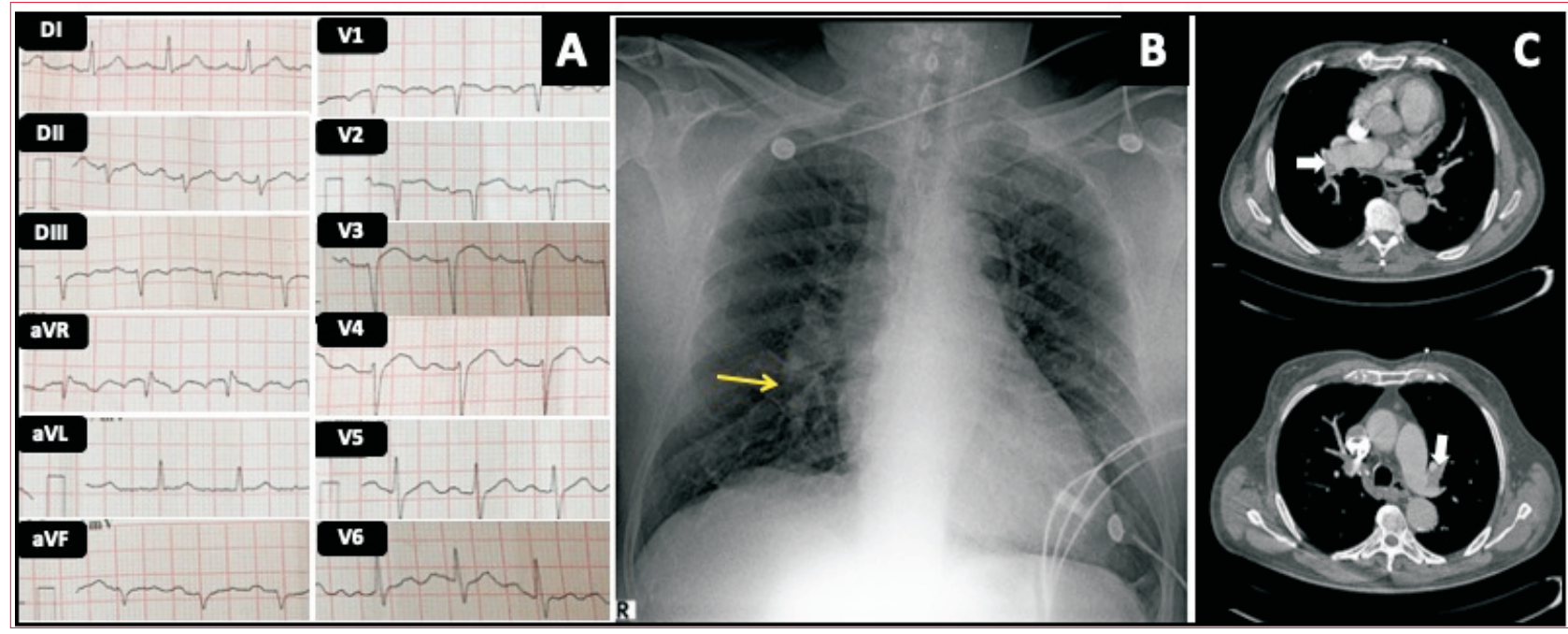

Figura 1. Caso clínico 1. A) ECG al ingreso que evidencia: taquicardia sinusal de $110 \mathrm{cpm}$, BAV 1 ${ }^{\text {er }}$ grado, onda $\mathrm{Q}$ en DIII y aVF, supradesnivel ST en V1-V3 máximo $2 \mathrm{~mm}$, transición del eje en precordiales a nivel de V5. B) RxTx: flecha amarilla señala amputación de arteria pulmonar lobar inferior derecha. C) Cortes angio TC que evidencian TEP central (las flechas blancas marcan trombo endoluminal en ramas principales de arteria pulmonar).

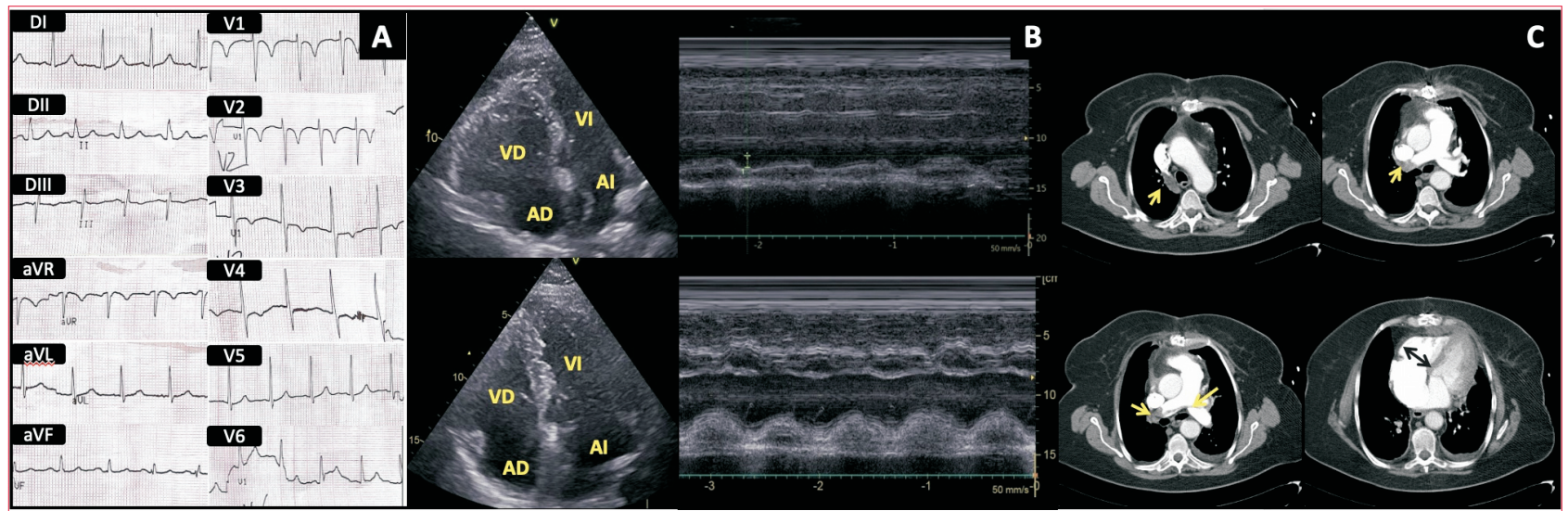

Figura 2. Caso clínico 2. A) ECG que evidencia taquicardia sinusal de 100 cpm, signos de HVI y ondas T invertidas de V1 a V4. B) Angio TC pulmonar: flechas amarillas indican defecto de relleno compatible con trombo en silla de montar que se extiende a ramas segmentarias. La doble flecha negra marca dilatación del ventrículo derecho (VD). En la misma imagen se observa desplazamiento del tabique interventricular hacia el ventrículo izquierdo (VI). C) Imagen ecocardiográfica 2D en vista apical 4 cámaras en diástole y excursión del anillo tricuspídeo mediante modo M. Arriba: imagen al diagnóstico que evidencia VD dilatado y disfuncionante (TAPSE: $12 \mathrm{~mm}$ ) y movimiento del septum interventricular hacia el VI. Abajo, imagen a las 24 h de la infusión de fibrinolíticos donde se observa reducción del diámetro basal del VD, franca mejora de su función (TAPSE: $16 \mathrm{~mm}$ ) y ausencia de desplazamiento del septum interventricular hacia el VI.

miento voluntario en domicilio por COVID-19 desde cuatro meses previos a la consulta. Consulta por ángor de reposo, de 20 minutos de duración, acompañado de disnea y SNV. ECG evidencia ritmo sinusal de $75 \mathrm{cpm}, \mathrm{P}$ y PR normales, QRS de $90 \mathrm{~ms}$ de duración con onda $\mathrm{Q}$ en DIII y ondas T invertidas en dicha derivación, sin otros trastornos de la repolarización. Curva de troponinas positiva y en ascenso (TnT $1^{\text {a }} 29,2^{\text {a }} 65$ y $3^{\text {a }} 98 \mathrm{pg} / \mathrm{ml}$, percentil 99:14 $\mathrm{pg} / \mathrm{ml})$. CACG evidencia estenosis no significativa en tercio proximal de arteria descendente anterior (FFR: 0,88), y estenosis crítica de tercio medio de arteria coronaria derecha (ACD). Se realiza angioplastia coronaria exitosa con implante de un stent liberador de everolimus sobre tercio medio de ACD (figuras 3A y 3B). A las 24 horas del procedimiento, al levantarse de la cama, presenta paro cardiorrespiratorio (PCR) en actividad eléctrica sin pulso. Se realizan maniobras de reanimación avanzada retomando circulación espontánea luego de 10 minutos. Normotenso, polipneico, saturando $94 \%$ con máscara de flujo libre, taquicárdico, sin trastorno de la conciencia y relleno capilar menor a $2 \mathrm{seg}$. ETT a los pies de la cama evidencia FEVI conservada, VD dilatado (basal $43 \mathrm{~mm}$ ) con función sistólica preservada, ausencia de derrame pericárdico. Gasometría post-PCR con insuficiencia respiratoria severa tipo 1 , lactato $4,3 \mathrm{mmol} / \mathrm{L}$. Se realiza angio TC que evi- 


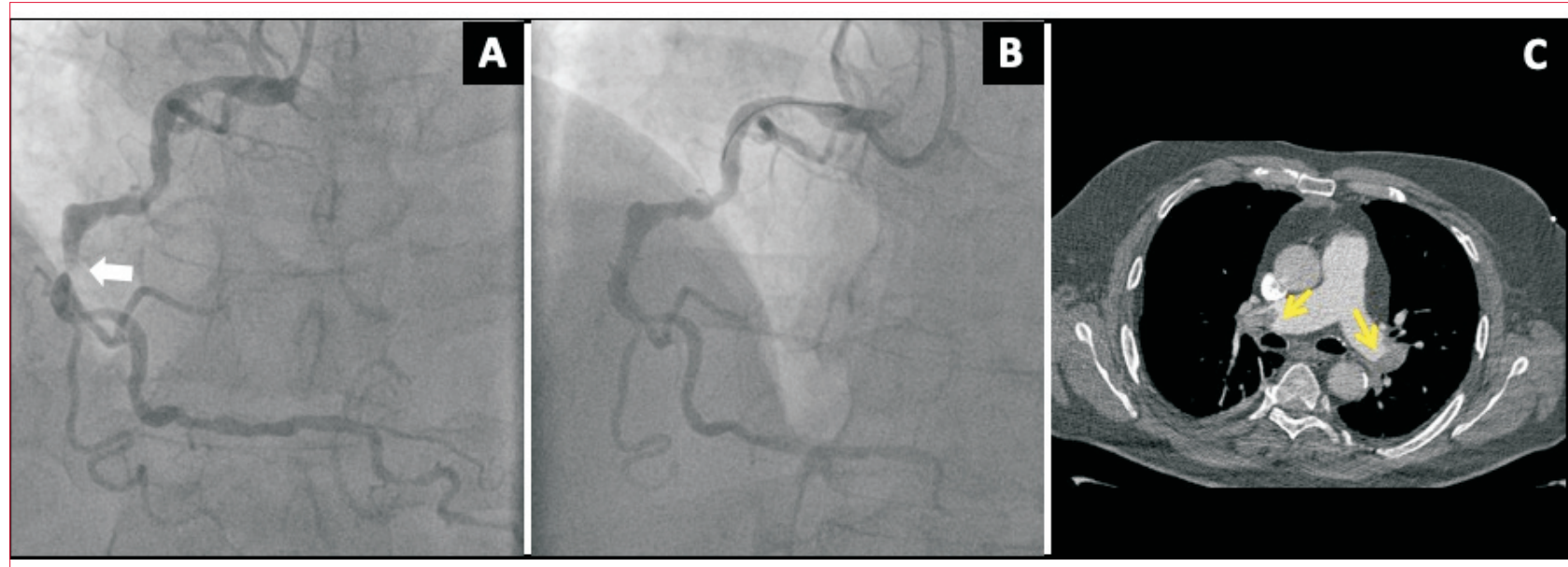

Figura 3. Caso clínico 3. A) Cateterización selectiva de la arteria coronaria derecha, donde se observa estenosis crítica de tercio medio (flecha blanca). B) Se observa cateterización selectiva de coronaria derecha luego de angioplastia exitosa de tercio medio con implante de stent liberador de everolimus 3,0 x $12 \mathrm{~mm}$. C) Angio TC pulmonar que evidencia defecto de relleno compatible con TEP central (flechas amarillas).

dencia TEP central con extensión hacia ramas segmentarias (figura 3C). Se decide administrar rTPA $100 \mathrm{mg} / \mathrm{iv}$ en dos horas, con buena tolerancia y rápida mejoría clínica y gasométrica, sin complicaciones hemorrágicas. Ecografía Doppler de miembros inferiores evidencia trombosis del sector distal de vena safena interna de miembro inferior derecho. Con buena evolución posterior se otorga el alta a domicilio a los diez días del ingreso bajo tratamiento con clopidogrel $75 \mathrm{mg}$ /día y apixabán $5 \mathrm{mg} / 12$ horas.

\section{Discusión}

La forma de presentación del TEP es muy variada e inespecífica, lo que resulta en subdiagnóstico o diagnóstico tardío. La alta sospecha clínica y la utilización de herramientas como las reglas de predicción clínica ${ }^{(6-8)}$ permiten aproximarnos a la probabilidad de TEP y disminuir el error diagnóstico.

Dentro de la sintomatología referida con más frecuencia por los pacientes con diagnóstico confirmado se destaca en una serie, relativamente reciente, la disnea en más del $70 \%$ de los casos, el dolor pleurítico en hasta $40 \%$ y la tos en $23 \%{ }^{(9)}$. El síncope se observó en 5\%-6\% de los casos, si bien este porcentaje es variable en las diferentes series, llegando en algunas de ellas a estar presente en uno de cada cuatro pacientes ${ }^{(9,10)}$. Es fundamental el alto índice de sospecha de TEP en sujetos que consultan por síncope, dado que se confirma el diagnóstico en hasta un $17 \%$ de los pacientes hospitalizados por este motivo ${ }^{(3)}$.

Se presentan tres casos de TEP grave con formas de presentación diferentes. En el caso 1, la clave para el diagnóstico estuvo en la alta sospecha clínica, poniendo de manifiesto que una correcta anamnesis devela una historia muy sugestiva. El ECG puede cons- tituir un factor confundidor, dado que los elementos de sufrimiento del VD pueden verse tanto en el TEP como en un evento coronario, resaltando nuevamente la importancia de la clínica para guiar el diagnóstico.

El caso clínico 2 representa un desafío diagnóstico inicial debido a las múltiples causas de síncope en un posoperatorio reciente de cirugía cardíaca. En esta situación el ETT fue la herramienta que permitió dilucidar el diagnóstico y descartar el taponamiento cardíaco como diferencial más importante.

Ambos casos se presentan inicialmente como síncope, lo cual se asocia fuertemente con inestabilidad hemodinámica y disfunción del VD (TEP grave) ${ }^{(11)}$.

Se citan tres grandes mecanismos de síncope asociados al TEP. El más frecuentemente reportado es la oclusión de la vasculatura pulmonar central, con falla aguda del VD, que determina reducción del gasto cardíaco con la consiguiente hipoperfusión cerebral que causa el síncope. En segundo lugar, el síncope podría deberse a un mecanismo arrítmico (por bradi o taquiarritmias), que podría originarse en la sobrecarga de volumen del VD. Por último, se ha propuesto que la oclusión de la vasculatura pulmonar podría desencadenar un reflejo vagal (Bezold-Jarisch), que genere una disminución brusca del gasto cardíaco y síncope neurocardiogénico ${ }^{(12-15)}$.

El caso clínico 3 se presentó inicialmente con ángor (observado hasta en $4 \%$ de los pacientes) ${ }^{(9)}$ e inversión de onda T en cara inferior, lo cual llevó a un error diagnóstico (síndrome coronario) y, por lo tanto, a un tratamiento de enfermedad coronaria concomitante. El dolor torácico en el contexto de TEP habitualmente es pleurítico, pero en el TEP central, que genera aumento de la poscarga del VD, puede determinar dolor anginoso. Concomitantemente, no debemos olvidar que la presencia de estenosis severas (más aún críti- 
cas) determina un menor umbral isquémico, lo que favorece la aparición de isquemia miocárdica ante el aumento de las demandas de oxígeno. El deterioro hemodinámico a las 24-48 horas del evento inicial podría ser explicado por un nuevo evento embólico o por una respuesta inflamatoria que afecta al VD, configurando una "miocarditis" del VD inducida por TEP ${ }^{(16)}$. El contexto del PCR en el caso que presentamos (luego de levantarse), podría sugerir el primer mecanismo.

En la tabla 1 se describen los principales hallazgos electrocardiográficos que pueden encontrarse en los pacientes con TEP confirmado. Estos se relacionan sobre todo con la aparición de cor pulmonar agudo. En una serie de 49 pacientes con TEP confirmado se detectó que $76 \%$ tenía tres o más de estos hallazgos, aunque hasta 26\% tenía un ECG normal al ingreso. Esto hace que el ECG presente baja sensibilidad y su rol preponderante sea sobre todo para diferenciar esta entidad de un $\mathrm{IM}^{(17,18)}$. En ninguno de los tres casos presentados se evidencian tres o más de los hallazgos mencionados.

En los tres casos se evidenció elevación del nivel de Tn por encima del P99. En el caso 1, además, se dosificó y se observó elevación del nivel de péptidos natriuréticos. Las primeras son marcadores de daño miocárdico, y los segundos indican disfunción ventricular derecha.. Ambos parámetros se han asociado a mayor número de complicaciones intrahospitalarias y muerte en los pacientes con $\operatorname{TEP}^{(19,20)}$. Sin embargo, los valores elevados de estos biomarcadores tienen un valor predictivo positivo bajo, pero un alto valor predictivo negativo, lo cual significa que con un valor por debajo del P99 aleja la posibilidad de complicaciones y muerte pre$\mathrm{coz}^{(21,22)}$. Por lo tanto, los biomarcadores podrían ser útiles en los pacientes de no alto riesgo.

El TEP de alto riesgo se define actualmente como aquel que presenta al menos uno de los siguientes criterios: paro cardíaco (caso 3), hipotensión persistente o shock obstructivo (casos 1 y 2$)^{(5)}$. El reconocimiento de este subgrupo de pacientes tiene importancia pronóstica (mayor mortalidad) y terapéutica. En cuanto a las medidas terapéuticas, el tratamiento fibrinolítico, combinado con la anticoagulación con heparina, ha demostrado disminuir la mortalidad y la recurrencia del TEP en pacientes de alto riesgo cuando se compara contra el tratamiento exclusivo con heparina (indicación de clase I) ${ }^{(23)}$. El mayor beneficio de los fibrinolíticos se observa cuando el tratamiento se inicia en las primeras 48 horas del inicio de los síntomas, aunque puede ser útil hasta los 14 días ${ }^{(24)}$. El tratamiento concomitante consiste en las medidas de sostén hemodinámico y respiratorio. El primero se basa en mejorar la precarga (aporte moderado de volumen) y eventual-
Tabla 1. Hallazgo electrocardlográficos frecuentes en el TEP ${ }^{(17)}$

BIRD o BCRD con elevación del ST $>1 \mathrm{~mm}$ y ondas $\mathrm{T}+$ en $\mathrm{V} 1$

Onda 5 en DI y aVL $>1,5 \mathrm{~mm}$

Transición en precordiales en V5

Ondas Q en DIII y aVF pero no en DII

Desviación del eje del QRS a derecha ( $\left.>90^{\circ}\right)$ o eje indeterminado

Voltaje del QR5 $<5 \mathrm{~mm}$ en todas las derivadas de los miembros

Ondas T invertidas en DIII y aVF o de V1 a V4

BIRD: bloqueo incompleto de rama derecha. BCRD: bloqueo completo de rama derecha.

mente apoyo inotrópico y vasopresor; el segundo se basa en oxigenoterapia y, de ser necesario, asistencia ventilatoria mecánica.

Después del tratamiento de reperfusión y la estabilización hemodinámica y respiratoria, la anticoagulación parenteral se puede cambiar por anticoagulación oral y ésta debe mantenerse al menos tres meses ${ }^{(25)}$, debiendo evaluarse posteriormente la presencia de factores de riesgo que predispongan a la recurrencia.

\section{Conclusión}

El diagnóstico de TEP puede ser complejo y debe existir siempre un alto índice de sospecha. Ante un paciente con síncope, siempre debe considerarse el TEP como causa debido a las implicancias pronósticas y terapéuticas; constituye un indicador probable de repercusión hemodinámica, aunque no exista hipotensión al momento de la consulta, y debe tomarse una conducta agresiva para un rápido diagnóstico y tratamiento. Es de fundamental utilidad contar con ecocardiograma a los pies de la cama para una rápida aproximación diagnóstica, ya que el inicio precoz del tratamiento anticoagulante y la reperfusión farmacológica en el subgrupo de alto riesgo constituyen las medidas con mayor impacto pronóstico.

Gimena Loza, http://orcid.org/0000-0001-9555-7431

Victoria Briano, https://orcid.org/0000-0001-8212-7715

Agustina Perdomo, https://orcid.org/0000-0003-4810-2383

Álvaro Niggemeyer, http://orcid.org/0000-0003-0911-6029

Juan Albistur, https://orcid.org/0000-0002-0282-5263

Este artículo fue aceptado para su publicación por el Dr. Federico Ferrando 


\section{Bibliografía}

1. Raskob GE, Angchaisuksiri P, Blanco AN, Buller H, Gallus A, Hunt BJ, et al. Thrombosis: a major contributor to global disease burden. Arterioscler Thromb Vasc Biol. noviembre de 2014;34(11):2363-71.

2. Wendelboe AM, Raskob GE. Global Burden of Thrombosis: Epidemiologic Aspects. Circ Res. 29 de abril de 2016;118(9):1340-7.

3. Prandoni P, Lensing AWA, Prins MH, Ciammaichella M, Perlati M, Mumoli N, et al. Prevalence of Pulmonary Embolism among Patients Hospitalized for Syncope. N Engl J Med. 20 de octubre de 2016;375 (16):1524-31.

4. Kucher N, Rossi E, De Rosa M, Goldhaber SZ. Massive pulmonary embolism. Circulation. 31 de enero de 2006;113(4):577-82.

5. Konstantinides SV, Meyer G, Becattini C, Bueno H, Geersing G-J, Harjola V-P, et al. 2019 ESC Guidelines for the diagnosis and management of acute pulmonary embolism developed in collaboration with the European Respiratory Society (ERS). Eur Heart J. 21 de enero de 2020;41(4):543-603.

6. Le Gal G, Righini M, Roy P-M, Sanchez O, Aujesky D, Bounameaux H, et al. Prediction of pulmonary embolism in the emergency department: the revised Geneva score. Ann Intern Med. 7 de febrero de 2006;144(3):165-71.

7. Gibson NS, Sohne M, Kruip MJHA, Tick LW, Gerdes VE, Bossuyt PM, et al. Further validation and simplification of the Wells clinical decision rule in pulmonary embolism. Thromb Haemost. enero de 2008;99(1):229-34.

8. Wells PS, Ginsberg JS, Anderson DR, Kearon C, Gent M, Turpie AG, et al. Use of a clinical model for safe management of patients with suspected pulmonary embolism. Ann Intern Med. 15 de diciembre de 1998;129(12):997-1005.

9. Pollack CV, Schreiber D, Goldhaber SZ, Slattery D, Fanikos J, O'Neil BJ, et al. Clinical characteristics, management, and outcomes of patients diagnosed with acute pulmonary embolism in the emergency department: initial report of EMPEROR (Multicenter Emergency Medicine Pulmonary Embolism in the Real World Registry). J Am Coll Cardiol. 8 de febrero de 2011;57(6):700-6.

10. Miniati M, Prediletto R, Formichi B, Marini C, Di Ricco G, Tonelli L, et al. Accuracy of clinical assessment in the diagnosis of pulmonary embolism. Am J Respir Crit Care Med. marzo de 1999;159(3):864-71.

11. Barco S, Ende-Verhaar YM, Becattini C, Jimenez D, Lankeit M, Huisman MV, et al. Differential impact of syncope on the prognosis of patients with acute pulmonary embolism: a systematic review and meta-analysis. Eur Heart J. 14 de diciembre de 2018;39(47):4186-95.
12. Wilner C, Garnier-Crussard JP, Huygue De Mahenge A, Gayet C, André-Fouet X, Pont M. [Paroxysmal atrioventricular block, cause of syncope in pulmonary embolism. 2 cases]. Presse Medicale Paris Fr 1983. 24 de diciembre de 1983;12(47):2987-9.

13. Keller K, Beule J, Balzer JO, Dippold W. Syncope and collapse in acute pulmonary embolism. Am J Emerg Med. julio de 2016;34(7):1251-7.

14. Thames MD, Alpert JS, Dalen JE. Syncope in patients with pulmonary embolism. JAMA. 5 de diciembre de 1977;238(23):2509-11.

15. Simpson RJJ, Podolak R, Mangano CAJ, Foster JR, Dalldorf FG. Vagal syncope during recurrent pulmonary embolism. JAMA. 21 de enero de 1983;249(3): 390-3.

16. Begieneman MPV, van de Goot FRW, van der Bilt IAC, Vonk Noordegraaf A, Spreeuwenberg MD, Paulus WJ, et al. Pulmonary embolism causes endomyocarditis in the human heart. Heart Br Card Soc. abril de 2008;94(4):450-6.

17. Sreeram N, Cheriex EC, Smeets JL, Gorgels AP, Wellens HJ. Value of the 12-lead electrocardiogram at hospital admission in the diagnosis of pulmonary embolism. Am J Cardiol. 1 de febrero de 1994;73(4): 298-303.

18. McIntyre KM, Sasahara AA, Littmann D. Relation of the electrocardiogram to hemodynamic alterations in pulmonary embolism. Am J Cardiol. agosto de 1972;30(3):205-10.

19. Klok FA, Mos ICM, Huisman MV. Brain-type natriuretic peptide levels in the prediction of adverse outcome in patients with pulmonary embolism: a systematic review and meta-analysis. Am J Respir Crit Care Med. 15 de agosto de 2008;178(4):425-30.

20. Becattini C, Vedovati MC, Agnelli G. Prognostic value of troponins in acute pulmonary embolism: a meta-analysis. Circulation. 24 de julio de 2007;116(4): 427-33.

21. Coutance G, Cauderlier E, Ehtisham J, Hamon M, Hamon M. The prognostic value of markers of right ventricular dysfunction in pulmonary embolism: a meta-analysis. Crit Care Lond Engl. 2011;15(2): R103.

22. Kucher N, Goldhaber SZ. Cardiac biomarkers for risk stratification of patients with acute pulmonary embolism. Circulation. 4 de noviembre de 2003;108(18): 2191-4.

23. Marti C, John G, Konstantinides S, Combescure C, Sanchez O, Lankeit M, et al. Systemic thrombolytic therapy for acute pulmonary embolism: a systematic review and meta-analysis. Eur Heart J. 7 de marzo de 2015;36(10):605-14.

24. Daniels LB, Parker JA, Patel SR, Grodstein F, Goldhaber SZ. Relation of duration of symptoms with res- 
ponse to thrombolytic therapy in pulmonary embolism. Am J Cardiol. 15 de julio de 1997;80(2):184-8.

25. Boutitie F, Pinede L, Schulman S, Agnelli G, Raskob G, Julian J, et al. Influence of preceding length of anticoagulant treatment and initial presentation of venous thromboembolism on risk of recurrence after stopping treatment: analysis of individual participants' data from seven trials. BMJ. 24 de mayo de 2011;342:d3036.

\section{Contribución de los autores}

Gimena Loza: redacción de casos clínicos, redacción de discusión, búsqueda bibliográfica, elaboración de imágenes, revisión y corrección del manuscrito.

Victoria Briano: redacción de casos clínicos, redacción de discusión, búsqueda bibliográfica.

Agustina Perdomo: redacción de casos clínicos, redacción de discusión, búsqueda bibliográfica.

Álvaro Niggemeyer: redacción de discusión, revisión y corrección del manuscrito

Juan Albistur: redacción de casos clínicos, redacción de discusión, búsqueda bibliográfica, elaboración de imágenes, revisión y corrección del manuscrito. 